\title{
Minimal Coordinate Formulation of Contact Dynamics in Operational Space
}

\author{
Abhinandan Jain, Cory Crean, Calvin Kuo, Steven Myint \\ Jet Propulsion Laboratory, California Institute of Technology \\ Pasadena, CA 91109
}

\author{
Hubertus von Bremen \\ California State Polytechnic University \\ Pomona, CA 91768
}

\begin{abstract}
In recent years, complementarity techniques have been developed for modeling non-smooth contact and collision dynamics problems for multi-link robotic systems. In this approach, a linear complementarity problem (LCP) is set up using $6 n$ non-minimal coordinates for a system with $n$ links together with all the unilateral constraints and inter-link bilateral constraints on the system. In this paper, we use operational space dynamics to develop a complementarity formulation for contact and collision dynamics that uses minimal coordinates. The use of such non-redundant coordinates results in much smaller size LCP problems and the automatic enforcement of the inter-link bilateral constraints. Furthermore, we exploit operational space low-order algorithms to overcome some of the computational bottlenecks in using minimal coordinates.
\end{abstract}

\section{INTRODUCTION}

For more than a decade, researchers [3, 11, 13] have been developing complementarity based approaches for formulating and solving the equations of motion of systems with contact and collision dynamics. Examples of such dynamics for robotic systems include manipulation and grasping tasks, and legged locomotion. This approach models bodies as rigid, and uses impulsive dynamics to handle non-smooth collision, contact interactions and state transitions. By essentially impulsively "stepping" over non-smooth events, complementarity methods avoid the the small step size and stiff dynamics problems encountered with penalty based methods which model surface deformation dynamics during contact [9].

The complementarity approach involves setting up of a linear complementarity problem ( $L C P$ ) that depends on the link mass and inertia properties, contact friction parameters, inter-link bilateral constraints and contact and collision unilateral constraints [3, 11, 13]. The LCP solution identifies the unilateral constraints that are active, and solves for the impulsive forces and velocity changes that are consistent with the constraints on the system. Variants of the complementarity approach to handle elastic and inelastic collisions have also been developed [3]. While the standard LCP formulation uses a discretized approximation for the friction cones, other researchers [12] have explored non-linear cone complementarity approaches that avoid such approximations.

For a multi-link system with $n$ links, the conventional LCP problem setup uses $6 n$ non-minimal coordinates [13] together with the bilateral constraints associated with the inter-link hinges and the unilateral contact constraints. This approach has a mass matrix that is block diagonal and constant. Such redundant coordinates lead to large LCP problem size, and require the use of differential-algebraic equation (DAE) like techniques for managing error drift in the bilateral constraints when integrating the equations of motion.

An alternative approach uses minimal hinge coordinates that automatically eliminate the bilateral constraints for the inter-link hinges [14]. While the underlying physics remains unchanged, this formulation reduces the size of the LCP problems, and avoids the need for DAE techniques for controlling bilateral constraint violation errors for the inter-link hinges. However, the use of minimal coordinate leads to a dense and configuration dependent mass matrix. This consequent complexity and computational setup expense for the LCP problem has been a significant hurdle in the use of such a minimal coordinate approach.

In this paper, we focus on the analytical and computational aspects of the minimal coordinate formulation of the complementarity approach to contact and collision dynamics for multi-link systems. We adopt the complementarity based physics models from [3, 13], but reformulate the system dynamics and the associated LCP problem using minimal coordinates. While [14] uses a similar minimal coordinate formulation, it is limited to just contact dynamics, and uses the non-optimal divide-and-conquer (DCA) technique [4] as part of its solution technique. This paper goes beyond contact dynamics to also develop the LCP variants for handling elastic and inelastic collision dynamics. Moreover, for closed-chain topology systems, we describe a uniform way to incorporate the bilateral constraints naturally into the LCP problem. The size of the resulting LCP problem is independent of the number of links and generalized coordinates, and only depends on the number of contact nodes. Our minimal coordinates approach adopts an operational space [7] perspective, which in turn allows us to take advantage of low-order spatial operator algorithms [5, 6, 8] for computing the operational space complementarity matrix (OSCM) needed for setting up the LCP problem. Taken together the methods described here provide a comprehensive, and computationally tractable, solution to using minimal coordinates for contact and collision dynamics problems. We conclude with an illustrative multilink pendulum numerical problem to benchmark the performance improvements from the minimal coordinates approach. 


\section{EQUATIONS OF Motion}

\section{A. Minimal coordinate dynamics}

The minimal coordinate equations of motion for a treetopology multi-link robotic system with $n$ links and $\mathcal{N}$ degrees of freedom has the form

$$
\mathcal{T}=\mathcal{M} \ddot{\theta}+\mathcal{C}-\mathcal{J}^{*} \mathfrak{f}_{\mathrm{c}}
$$

where $\theta \in \mathcal{R}^{\mathcal{N}}$ denotes the generalized coordinates, $\mathcal{T} \in \mathcal{R}^{\mathcal{N}}$ the generalized forces, $\mathcal{N} \in \mathcal{R}^{\mathcal{N} \times \mathcal{N}}$ the system mass matrix, $\mathcal{C} \in \mathcal{R}^{\mathcal{N}}$ the vector of Coriolis, gyroscopic and gravitational forces, $\mathfrak{f}_{c} \in \mathcal{R}^{6 n_{c}}$ the stacked vector of nodal forces for $n_{c}$ nodes on the system, and $\mathcal{J} \in \mathcal{R}^{6 n_{c} \times \mathcal{N}}$ the Jacobian matrix for these nodes. In a minimal coordinates formulation, bilateral constraints associated with inter-link hinges are eliminated by using hinge coordinates that directly parameterize the permissible hinge motion. Thus minimal coordinate models of tree-topology systems have no bilateral constraints.

Bilateral constraints on the other hand are unavoidable even when using minimal coordinates for closed-chain topology systems. Such systems are decomposed into a tree-topology system (formed by a spanning tree) together with a minimal set of bilateral closure constraints. While the closure constraints define the bilateral constraints, contact constraints lead to a set of unilateral constraints on the system.

While the values of the $\mathfrak{f}_{\mathrm{c}}$ nodal force components that arise from control actuators may be known, the ones associated with system constraints are not explicitly available. In this paper, we will assume that $\mathfrak{f}_{\mathfrak{c}}$ only contains such implicit constraint forces, since the effect of the explicit ones is easily handled by absorbing them into $\mathcal{C}$. In view of this, all nodes in this paper will refer to constraint nodes. With $\mathfrak{f}_{c}$ denoting the implicit constraint forces, Eq. 1 extends to also be the smooth equations of motion for non-tree systems,

The $\ddot{\theta}$ accelerations solution of Eq. 1 can be expressed as

$$
\ddot{\theta}=\mathcal{M}^{-1} \mathfrak{g}^{*} \mathfrak{f}_{\mathrm{c}}+\ddot{\theta}_{\mathrm{f}} \text { where } \ddot{\theta}_{\mathrm{f}} \triangleq \mathcal{M}^{-1}(\mathcal{T}-\mathcal{C})
$$

$\ddot{\theta}_{f}$ represents the free generalized accelerations, i.e. the generalized accelerations in the absence of the nodal forces. Eq. 2 expresses the overall $\ddot{\theta}$ generalized acceleration for the system as the sum of the $\ddot{\theta}_{f}$ free generalized accelerations and the $\mathcal{M}^{-1} \mathcal{J}^{*} \mathfrak{f}_{\mathrm{c}}$ correction acceleration contribution from the implicit non-zero nodal forces. The next section describes the operational space form of the equations of motion that describe the mapping between the nodal forces and the nodal spatial accelerations.

\section{B. Operational space dynamics}

The operational space for the multi-link system is defined by the configuration of the set of constraint nodes on the system. Let $\mathcal{V}_{\mathrm{c}} \in \mathcal{R}^{6 \mathrm{n}_{\mathrm{c}}}$ denote the stacked vector of spatial velocities [6] of the $n_{c}$ nodes. The relationship between $\mathcal{V}_{c}$ and the $\dot{\theta}$ joint velocities is given by

$$
\mathcal{V}_{\mathrm{c}}=\dot{\partial} \dot{\theta}
$$

The constraint nodes spatial acceleration $\alpha_{c} \in \mathcal{R}^{6 n_{c}}$ is the time derivative of $\mathcal{V}_{c}$ which, from Eq. 3 is given by

$$
\alpha_{\mathrm{c}} \triangleq \frac{\mathrm{d} \mathcal{V}_{\mathrm{c}}}{\mathrm{dt}} \stackrel{3}{\underline{\underline{3}}} \ddot{\partial}+\mathfrak{a}_{\mathrm{os}} \quad \text { with } \quad \mathfrak{a}_{\mathrm{os}} \triangleq \dot{\mathrm{j} \dot{\theta}}
$$

Denoting the node accelerations for the free system (i.e. without the $\mathfrak{f}_{\mathrm{c}}$ nodal forces) by $\alpha_{\mathrm{f}}$, the following analog of Eq. 4 defines $\alpha_{f}$ in terms of the $\ddot{\theta}_{f}$ generalized accelerations:

$$
\alpha_{f} \triangleq \partial \ddot{\theta}_{f}+\mathfrak{a}_{\mathrm{os}}
$$

Pre-multiplying Eq. 2 by $\mathcal{J}$ and using Eq. 4 yields:

$$
\alpha_{c} \stackrel{\sqrt[45.52]{=}}{=} \underline{\Lambda} \mathfrak{f}_{\mathrm{c}}+\alpha_{\mathrm{f}} \text { where } \underline{\Lambda} \triangleq \partial \mathcal{M}^{-1} \mathrm{~J}^{*} \in \mathcal{R}^{6 \mathrm{n}_{\mathrm{c}} \times 6 \mathrm{n}_{\mathrm{c}}}
$$

$\Lambda$ is referred to as the operational space compliance matrix $(O S C M)$. The invertibility of $\underline{\Lambda}$ does not depend on $\mathcal{J}$ being invertible - only that $\mathcal{J}$ have full row-rank. When it exists, the inverse of $\underline{\Lambda}$ is referred to as the operational space inertia.

\section{Impulsive dynamics}

The differential form of the equations of motion in Eq. 2 can be discretized using an Euler step to obtain a form that maps a $p$ impulse stacked vector at the nodes (over a $\Delta_{t}$ time interval) into the resulting change in generalized velocities 1

$$
\dot{\theta}^{+}-\dot{\theta}^{-} \stackrel{2}{\underline{2}} \mathcal{M}^{-1} g^{*} p+\ddot{\theta}_{\mathrm{f}} \Delta_{\mathrm{t}} \text { with } p \triangleq \mathfrak{f}_{\mathrm{c}} \Delta_{\mathrm{t}}
$$

Multiplying both sides with $\mathcal{J}$ leads to the following expression for the change in nodal spatial velocities

$$
V_{c}^{+}-V_{c}^{-} \stackrel{3,7}{=} \underline{\underline{p}} \underline{\underline{p}}+\alpha_{\mathrm{f}} \Delta_{\mathrm{t}}
$$

Collision events are impulsive and lead to instantaneous changes in the system velocities, and in this case $\Delta_{t}=0$ in the above equation.

\section{UNILATERAL CONTACT CONSTRAINTS}

Unilateral constraints are defined by inequality relationships of the form

$$
\mathfrak{d}(\theta, \mathrm{t}) \geqslant 0
$$

As an example, the non-penetration condition for rigid bodies can be stated as an inequality relationship requiring that the distance between the surfaces of rigid bodies be non-negative. $\mathfrak{d}(\theta, t)$ is generally referred to as the distance or gap function for unilateral constraints.

Contact occurs at the constraint boundary, i.e., when $\mathfrak{d}(\theta, t)=0$. For bodies in contact, the surface normals at the contact point are parallel. The existence of contact is typically determined using geometric or collision detection techniques. For a pair of bodies $A$ and $B$ in contact, we use a convention where the $i^{\text {th }}$ contact normal $\hat{n}(i)$ is defined as pointing from body $B$ towards body $A$, so that motion of $A$ in the direction of the normal leads to a separation of the bodies.

A unilateral constraint is said to be in an active state when

$$
\mathfrak{d}(\theta, \mathfrak{t})=\dot{\mathfrak{d}}(\theta, \mathrm{t})=\ddot{\mathfrak{d}}(\theta, \mathrm{t})=0
$$

\footnotetext{
${ }^{1}$ The - and + superscripts denote the respective value of a quantity just before and after the application of an impulse.
} 
Thus, a unilateral constraint is active when there is contact, and the contact persists. Only active constraints generate constraint forces on the system.

A constraint that is not active is said to be inactive. Contact separation occurs when the relative linear velocity of the contact points along the normals becomes positive and the contact points drift apart. A separating constraint is in the process of losing contact and transitioning to an inactive state, and at the start of a separation event, we have

$$
\mathfrak{d}(\theta, t)=\dot{\mathfrak{d}}(\theta, \mathrm{t})=0 \quad \text { and } \quad \ddot{\mathfrak{d}}(\theta, \mathrm{t})>0
$$

\section{A. Contact impulse for an active contact constraint}

We now describe contact force modeling using the approach in references [3, 13]. Denote the number of unilateral contact nodes by $n_{u}$. The 6 -dimensional spatial impulse at the $i^{\text {th }}$ active contact constraint node has a zero angular moment component. Its non-zero linear impulse component $F_{\mathfrak{u}}(i) \in \mathcal{R}^{3}$ can be decomposed into normal and tangential (friction impulse) components

$$
F_{u}(\mathfrak{i})=F_{n}(i) \hat{n}(i)+F_{t}(i) \hat{t}(i)
$$

$\hat{t}(i)$ denotes a tangent plane vector for the $i^{\text {th }}$ contact pair. Assuming that the friction coefficient is $\mu(i)$, the magnitude of the tangential Coulomb frictional impulse is bounded by the magnitude of the normal component as follows:

$$
\left\|F_{t}(i)\right\| \leqslant \mu(i) F_{n}(i)
$$

When the relative linear velocity between the contact nodes is non-zero, the tangential frictional impulse is in a direction opposing the linear velocity vector (which necessarily lies in the contact tangent plane) and Eq. 13 holds with an equality. When the bodies have non-zero relative linear velocities at the contact point, the contact is said to be a sliding contact. Otherwise, when the relative linear velocity is zero, the contact is said to be a rolling contact. Thus, the tangential friction impulse is on the boundary of the cone defined by Eq. 13 when sliding, and in the interior of the cone when rolling.

For the purpose of numerical computation, the friction cone at the $i^{\text {th }}$ contact is approximated by a friction polyhedron consisting of a finite number, $n_{f}$, of unit direction vectors $\hat{d}_{\mathfrak{j}}(i)$ in the tangent plane. It is assumed that for each direction vector, its opposite direction vector is also in the set. For notational simplicity, we assume that $n_{f}$ is the same across all contact points. The $i^{\text {th }}$ contact tangential frictional impulse is expressed as the linear combination of these direction vectors as follows:

$$
\begin{aligned}
F_{t}(i) \hat{t}(i) & =\sum_{j=0}^{n_{f}} \beta_{j}(i) \hat{d}_{j}(i)=D(i) \beta(i) \\
\text { where } D(i) & \triangleq\left[\hat{d}_{1}(i), \cdots, \hat{d}_{n_{f}}(i)\right] \in \mathcal{R}^{3 \times n_{f}} \\
\text { and } \beta(i) & \triangleq \operatorname{col}\left\{\beta_{j}(i)\right\}_{j=1}^{n_{f}} \in \mathcal{R}^{n_{f}}
\end{aligned}
$$

Combining Eq. 12 and Eq. 14 we have

$$
\begin{gathered}
F_{u}(i)=\underline{D}(i) \underline{\beta}(i) \text { where } \underline{\beta}(i) \triangleq\left[\begin{array}{c}
F_{n}(i) \\
\beta(i)
\end{array}\right] \in \mathcal{R}^{n_{f}+1} \\
\text { and } \underline{D}(i) \triangleq[\hat{n}(i), D(i)] \in \mathcal{R}^{3 \times\left(n_{f}+1\right)}
\end{gathered}
$$

During sliding, the $\beta_{j}(i)$ component is non-zero and equal to $\mu(i) F_{n}(i)$ for just the single $j$ that corresponds to the closest direction opposing the (tangential) relative linear velocity. In other words, with $\sigma(i)$ denoting the magnitude of the contact relative linear velocity,

$$
\beta_{k}(i)=\left\{\begin{array}{lll}
\mu(i) F_{n}(i) \mathbb{1}_{[k=j]} & \text { if } & \sigma(i)>0 \\
0 & \text { if } & \sigma(i)=0
\end{array}\right.
$$

In the above, $\mathbb{1}_{[<\text {cond }>]}$ denotes the indicator function whose value is 1 if the condition is true, and 0 otherwise.

\section{B. Complementarity relationship for a unilateral contact}

The sliding/rolling contact relationships can be stated equivalently as the following standard complementarity conditions $\Omega^{2}$

$$
\begin{array}{rrrr}
\hat{n}^{*}(i) \nu_{u}^{+}(i) & \perp F_{n}(i) & \text { (separation) } \\
\sigma(i) E(i)+D^{*}(i) \nu_{u}^{+}(i) & \perp \beta(i) & \text { (friction force direction) } \\
\mu(i) F_{n}(i)-E^{*}(i) \beta(i) & \perp \sigma(i) & \text { (friction force magnitude) }
\end{array}
$$

$$
\text { where } E(i)=\operatorname{col}\{1\}_{j=1}^{n_{f}} \in \mathcal{R}^{n_{f}}
$$

$v_{u}^{+}(i) \in \mathcal{R}^{3}$ denotes the linear relative velocity of the first body $A$ with respect to the second body $B$. The component of this relative velocity along the contact normal is, $\hat{n}^{*}(\mathfrak{i}) \nu_{u}^{+}(\mathfrak{i})$, and when positive, indicates increasing separation between the bodies, while a negative value indicates that the bodies are approaching each other. The complementarity conditions in Eq. 17 enforce the no inter-penetration constraint at the velocity instead of at the gap level. Hence they are valid only when the gap is zero, i.e., when contact exists [3]. Using Eq. 15 , Eq. 17 can be alternatively expressed as

$$
\begin{aligned}
& \hat{\mathrm{E}}(\mathfrak{i}) \sigma(\mathfrak{i})+\underline{\mathrm{D}}^{*}(\mathrm{i}) \nu_{\mathfrak{u}}^{+}(\mathrm{i}) \perp \underline{\beta}(\mathrm{i}) \\
& \overline{\mathrm{E}}(i) \underline{\beta}(i) \perp \sigma(i) \\
& \text { where } \hat{E}(i) \triangleq\left[\begin{array}{c}
0 \\
E(i)
\end{array}\right] \in \mathcal{R}^{n_{f}+1} \\
& \text { and } \quad \overline{\mathrm{E}}(\mathfrak{i}) \triangleq\left[\mu(\mathfrak{i}),-\mathrm{E}^{*}(\mathfrak{i})\right] \in \mathcal{R}^{1 \times \mathfrak{n}_{\mathrm{f}}+1}
\end{aligned}
$$

\footnotetext{
${ }^{2}$ A complementarity condition, $f(x) \perp x$, holds for a function $\mathrm{f}(\mathrm{x}) \in \mathcal{R}^{\mathrm{n}}$ of a vector $x \in \mathcal{R}^{n}$, whose $x_{i}$ elements have lower and upper bounds $l_{i}$ and $u_{i}$ respectively, when the following properties apply: (a) $f_{i}(x) \geqslant 0$ when $x_{i}=l_{i}$; (b) $f_{i}(x) \leqslant 0$ when $x_{i}=u_{i}$; and (c) $f_{i}(x)=0$ when $l_{i}<x_{i}<u_{i}$. For the standard complementarity condition, $l_{i}=0$ and $u_{i}=\infty$. It is a linear complementarity condition when $f(x)$ has the form $M(x) x+q(x)$, and a mixed complementarity condition when $l_{i}=-\infty$ and $u_{i}=\infty$ for one or more of the elements.
} 


\section{Aggregated complementarity relationships}

For now we assume that there are no bilateral constraints on the system, and thus $n_{c}=n_{u}$ The stacked vector of relative linear velocities at the contacts is denoted $v_{u} \in \mathcal{R}^{3 n_{u}}$. It is related to the stacked vector of node spatial velocities $\nu_{c} \in$ $\mathcal{R}^{6 n_{c}}$ via the following

$$
v_{\mathfrak{u}}=Q_{u} \mathcal{V}_{\mathrm{c}}
$$

where the $Q_{\mathfrak{u}} \in \mathcal{R}^{3 n_{u} \times 6 n_{c}}$ matrix contains one block-row per contact node-pair, with each row mapping the spatial velocities of the node pair into the relative linear velocity across the contact. Defining and using the $Q_{\mathfrak{u}}$ matrix will later allow us to include unilateral constraints in a manner similar to bilateral constraints in the operational space formulation. $Q_{u}$ has the same structure as the $Q_{b}$ constraint mapping matrix for bilateral constraints described later in Eq. 32 for three degree of freedom spherical hinges.

The $\mathcal{Q}_{\mathfrak{u}}$ matrix also relates the $F_{\mathfrak{u}} \in \mathcal{R}^{3 n_{u}}$ equal and opposite impulses at contact node-pairs with the corresponding spatial impulses at the nodes, $p \in \mathcal{R}^{6 n_{c}}$, via

$$
p=Q_{u}^{*} F_{u}
$$

Define the stacked vectors

$$
\begin{aligned}
\underline{\beta} & \triangleq \operatorname{col}\{\underline{\beta}(i)\}_{i=0}^{n_{u}} \in \mathcal{R}^{n_{u}\left(n_{f}+1\right)} \\
\text { and } \quad \sigma & \triangleq \operatorname{col}\{\sigma(i)\}_{i=0}^{n_{u}} \in \mathcal{R}^{n_{u}}
\end{aligned}
$$

From Eq. 15, we have

$$
\begin{aligned}
\mathrm{F}_{\mathrm{u}} & \triangleq \operatorname{col}\left\{\mathrm{F}_{\mathrm{u}}(\mathrm{i})\right\}_{i=1}^{n_{u}}=\underline{\mathrm{D}} \underline{\beta} \in \mathcal{R}^{3 n_{u}} \\
\text { where } \quad \underline{\mathrm{D}} & \triangleq \operatorname{diag}\{\mathrm{D}(\mathrm{i})\}_{i=1}^{n_{u}} \in \mathcal{R}^{3 n_{u} \times n_{u}\left(n_{\mathrm{f}}+1\right)}
\end{aligned}
$$

Thus,

$$
p \stackrel{22 \underline{224}}{=} Q_{u}^{*} \underline{D} \underline{\beta}
$$

Now we examine the effect of the contact node impulses on the node spatial velocities.

$$
\nu_{c}^{+}-\nu_{c}^{-} \stackrel{\underline{\underline{8}}}{=} \underline{p}+\Delta_{\mathrm{t}} \alpha_{\mathrm{f}} \stackrel{\underline{24}}{=} \underline{\Lambda} \mathcal{Q}_{\mathfrak{u}}^{*} \underline{\underline{\beta}} \underline{\beta}+\Delta_{\mathrm{t}} \alpha_{\mathrm{f}}
$$

Pre-multiplying Eq. 26 by $\underline{D}^{*} Q_{u}$ we obtain

$$
\underline{D}^{*} v_{u}^{+} \stackrel{21}{=}\left(\underline{D}^{*} Q_{u} \underline{\Lambda} Q_{\mathfrak{u}}^{*} \underline{D}\right) \underline{\beta}+\Delta_{\mathrm{t}} \underline{D}^{*} Q_{u} \alpha_{\mathrm{f}}+\underline{D}^{*} v_{\mathrm{u}}^{-}
$$

Combining equations 19 and 27, the overall complementarity conditions can be rephrased as:

$$
\begin{gathered}
\left(\begin{array}{cc}
\underline{D}^{*} Q_{\mathfrak{u}} \frac{\Lambda}{\bar{E}} Q_{\mathfrak{u}}^{*} \underline{\mathrm{E}} & \hat{\mathrm{E}} \\
0
\end{array}\right)\left[\begin{array}{l}
\underline{\beta} \\
\sigma
\end{array}\right]+\left[\begin{array}{c}
\underline{D}^{*}\left(Q_{\mathfrak{u}} \Delta_{\mathrm{t}} \alpha_{\mathrm{f}}+v_{\mathrm{u}}^{-}\right) \\
0
\end{array}\right] \\
\perp\left[\frac{\beta}{\sigma}\right]
\end{gathered}
$$

where $\hat{E} \triangleq \operatorname{diag}\{\hat{E}(i)\}_{i=1}^{n_{u}} \in \mathcal{R}^{n_{u}\left(n_{f}+1\right) \times n_{u}}$ $\overline{\mathrm{E}} \triangleq \operatorname{diag}\{\overline{\mathrm{E}}(i)\}_{i=1}^{n_{u}} \in \mathcal{R}^{n_{u} \times n_{u}\left(n_{f}+1\right)}$
The structure of the standard LCP problem in Eq. 28 is different from that normally found in literature [3, 13, 14]. This difference arises from the way we have defined $\beta(i)$ in Eq. 15 and used it to arrange the coordinates in the LCP problem. The combined organization of the coordinates on a per contact constraint allows us to handle unilateral and bilateral constraints in a uniform manner. The difference is only structural and not in the underlying physics of the problem. The solution to this $n_{u}\left(n_{f}+2\right)$ dimensional LCP yields $\beta$ and $\sigma$, and these can be used to propagate the system state as follows:

$$
\begin{aligned}
p & =Q_{\mathfrak{u}}^{*} \underline{D} \underline{\beta} \\
\mathfrak{f}_{\mathrm{c}} & =p / \Delta_{\mathrm{t}} \\
\ddot{\theta} & =\ddot{\theta}_{\mathrm{f}}+\mathcal{M}^{-1} \mathfrak{J}^{*} \mathfrak{f}_{\mathrm{c}}
\end{aligned}
$$

The second equation can be numerically integrated until there is a new collision event during the $\Delta_{\mathrm{t}}$ propagation interval. Observe that the $p$ contact impulse is averaged over the $\Delta_{t}$ time interval to get the $\mathfrak{f}_{c}$ spatial forces at the contact nodes. An Euler step integration based discretization of Eq. 30 is:

$$
\dot{\theta}^{+}=\dot{\theta}^{-}+\mathcal{M}^{-1} \mathcal{f}^{*} p+\Delta_{t} \ddot{\theta}_{f}
$$

An LCP solution with $F_{\mathcal{u}}(i)$ positive indicates that the $i^{\text {th }}$ contact is active. Furthermore, a zero $\sigma(i)$ implies that the $i^{\text {th }}$ contact is a rolling contact while a non-zero value implies that it is a sliding contact.

\section{Including bilateral constraints}

As discussed earlier, in the minimal coordinate formulation, bilateral constraints arise from loop closure constraints in closed-chain topology systems. We now describe extensions to the complementarity formulation when the system has such bilateral constraints, and thus now $n_{c} \geqslant n_{u}$.

Assuming that $n_{\mathrm{b}}$ denotes the dimension of the bilateral constraints on the system, a bilateral constraint matrix $Q_{\mathrm{b}} \in$ $\mathcal{R}^{n_{b} \times 6 n_{c}}$ can be used to characterize the loop constraints as follows:

$$
\mathcal{Q}_{\mathrm{b}} \mathcal{V}_{\mathrm{c}}=0
$$

Eq. 22 and Eq. 25 for the node impulses generalize to

$$
p=Q_{u}^{*} F_{\mathfrak{u}}+Q_{\mathfrak{b}}^{*} \lambda \stackrel{24}{=} Q_{\mathfrak{u}}^{*} \underline{\underline{\beta}}+Q_{\mathrm{b}}^{*} \lambda
$$

where $\lambda \in \mathcal{R}^{\mathfrak{n}_{\mathrm{b}}}$ denotes the Lagrange multipliers associated with the bilateral constraints. $p$ now contains contributions from the unilateral as well as the bilateral constraints.

To handle the bilateral constraints, $\underline{\Lambda}$ in Eq. 27 expands to be the OSCM for the combined set of unilateral and bilateral constraint nodes. Using Eq. 33 in place of Eq. 25, the generalization of the complementarity condition in Eq. 28 
to include bilateral constraints is as follows:

$$
\begin{aligned}
& w=\mathfrak{M} z+q \quad \perp \quad z
\end{aligned}
$$

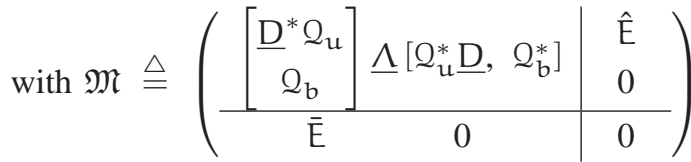

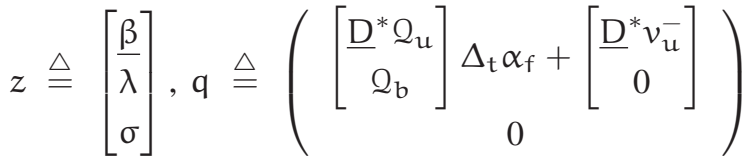

Here $\mathfrak{M}$ is a $\left(n_{u}\left(n_{f}+2\right)+n_{b}\right)$ size square matrix with $z$ and $q$ being $\left(n_{u}\left(n_{f}+2\right)+n_{b}\right)$ size vectors. The middle $\lambda$ row corresponds to the bilateral constraints, and its left hand side is required to be exactly zero to satisfy the algebraic bilateral constraints. Thus this represents a mixed LCP, with the lower bounds on the $\lambda$ variables being $-\infty$. Analogous to Eq. 31 , the solution to this LCP can be used to propagate the system state as follows:

$$
\begin{aligned}
p & =Q_{\mathfrak{u}}^{*} \underline{D} \underline{\beta}+Q_{\mathfrak{b}}^{*} \lambda \\
\dot{\theta}^{+} & =\dot{\theta}^{-}+\mathcal{M}^{-1} \mathcal{J}^{*} p+\Delta_{t} \ddot{\theta}_{f}
\end{aligned}
$$

\section{COLLISION DYNAMICS}

During inelastic collisions some of the impact energy is lost. The coefficient of restitution, $\epsilon(i)$ defines the fraction of the energy that remains after a collision. The complementarity approach to modeling collisions breaks up the collision event into instantaneous compression and decompression phases [3]. During the compression phase, collision energy is stored, and during decompression, a fraction of the collision impulse is recovered.

\section{A. Compression}

At the $i^{\text {th }}$ contact undergoing collision, the compression phase is instantaneous and impulsively changes the relative linear contact velocity from $v_{\mathfrak{u}}^{-}(\mathfrak{i})$ to a new $v_{\mathrm{c}}^{+}(\mathfrak{i})$ value with a non-negative normal component. The compression impulse is denoted $p_{c}(i)$. The mixed LCP problem for the compression phase is obtained by setting $\Delta_{\mathrm{t}}=0$ in Eq. 34 to obtain

$$
w=\mathfrak{M} z+\mathrm{q}_{\mathrm{c}} \perp z \quad \text { with } \quad \mathrm{q}_{\mathrm{c}} \triangleq\left(\left[\begin{array}{c}
{\left[\underline{\mathrm{D}}^{*} v_{\mathrm{u}}^{-}\right.} \\
0 \\
0
\end{array}\right]\right)
$$

The LCP solution is used to instantaneously (i.e. impulsively) propagate the state for the compression phase as follows:

$$
\begin{aligned}
p_{c} & =Q_{\mathfrak{u}}^{*} \underline{D} \underline{\beta}+Q_{\mathfrak{b}^{*}} \lambda \\
\dot{\theta}^{c} & =\dot{\theta}^{-}+\mathcal{M} \mathcal{M}^{-1} \partial^{*} p_{c} \\
v_{c}^{+} & =\mathcal{\partial} \dot{\theta}^{c}
\end{aligned}
$$

\section{B. Decompression}

The decompression phase applies a impulse of magnitude $\epsilon(i) \hat{n}^{*}(i) p_{c}(i)$ for the $i^{\text {th }}$ contact along the normal using the impulse stored during the compression phase. An extra contact impulse term ensures that the normal component of the relative linear velocity at the end of the decompression step remains non-negative. The recovered $\vartheta$ decompression impulse as

$$
\vartheta \triangleq \operatorname{col}\left\{\left(\epsilon(i) \hat{n}^{*}(i) p_{c}(i)\right) \hat{n}(i)\right\}_{i=1}^{n_{u}} \in \mathcal{R}^{3 n_{u}}
$$

The decompression phase LCP has the form:

$$
\begin{aligned}
& w=\mathfrak{M} z+q_{d} \quad \perp \quad z \\
& \text { with } \left.\mathrm{q}_{\mathrm{d}} \triangleq\left(\begin{array}{c}
{\left[\underline{\mathrm{D}}^{*} v_{\mathrm{c}}^{+}\right.} \\
0
\end{array}\right]+\underset{0}{\left[\begin{array}{c}
\underline{D}^{*} Q_{\mathfrak{u}} \\
Q_{\mathrm{b}}
\end{array}\right] \underline{\Lambda} \underline{Q}_{\mathrm{u}}^{*} \vartheta}\right)
\end{aligned}
$$

The decompression LCP problem is the mixed LCP in Eq. 34 with $\Delta_{\mathrm{t}}=0$, the contact linear velocity $v_{\mathrm{u}}^{-}$replaced with $v_{\mathrm{c}}^{+}$, and an additional $\left[\begin{array}{c}\underline{\mathrm{D}}^{*} Q_{\mathfrak{u}} \\ \mathcal{Q}_{\mathrm{b}}\end{array}\right] \underline{\Lambda} \underline{Q}_{\mathfrak{u}}^{*} \vartheta$ term for the recovered impulse in the q LCP vector term. The LCP solution is used to instantaneously propagate the state for the decompression phase as follows:

$$
\begin{aligned}
p & =Q_{\mathfrak{u}}^{*} \underline{\underline{\beta}}+Q_{\mathfrak{b}}^{*} \lambda+Q_{\mathfrak{u}}^{*} \vartheta \\
\dot{\theta}^{+} & =\dot{\theta}^{c}+\mathcal{M}^{-1} \jmath^{*} p
\end{aligned}
$$

When $\epsilon(i)=0$, there is no decompression phase. For collision dynamics, the numerical state propagation process consists of the following steps:

1) Use Eq. 35 to obtain new $\left[\theta\left(t+\Delta_{t}\right), \dot{\theta}\left(t+\Delta_{t}\right)\right]$ from the $[\theta(t), \dot{\theta}(t)]$ state.

2) If the new $\left[\theta\left(t+\Delta_{t}\right), \dot{\theta}\left(t+\Delta_{t}\right)\right]$ state involves a new collision, then estimate the $\Delta_{t}$ time for collision, and redo Step (1) with this new time interval. This should bring the system into contact for the new collision. Now use the compression and decompression LCPs in Eq. 37 and Eq. 40 to propagate the system through the collision.

An alternative to this discrete event state propagation approach is a time-stepping one, where the LCPs are solved only at the end of fixed time steps. This approach is faster, but at the cost of allowing some inter-penetration errors among colliding bodies.

\section{SPATIAl OPERATOR COMPUTATIONAL ALGORITHM}

The key implementation and computational challenge with using minimal coordinates is the need for computing $\underline{\Lambda}$ needed for setting up and solving the LCP problems in Eq. 28, Eq. 34, Eq. 36 and Eq. 39. As seen in Eq. 6, $\underline{\Lambda}$ involves the configuration dependent matrix products of the Jacobian matrix and the mass matrix inverse. A direct evaluation of this expression requires $\mathrm{O}\left(\mathcal{N}^{3}\right)$ computations. However references $[5,6,8]$ have used spatial operators to develop simpler and recursive computational algorithms for $\Lambda$ that are of only $\mathrm{O}(\mathcal{N})$ complexity. We briefly sketch out below the underlying analysis and structure of this algorithm, and refer the reader to $[5,6,8]$ for notation and derivation details. 


\section{A. Spatial operator factorization of $\mathcal{M}^{-1}$}

We begin with the following key spatial operator based analytical results that provide explicit, closed-form expressions for the factorization and inversion of a tree mass matrix [6, 10]:

$$
\begin{aligned}
\mathcal{N} & =\mathrm{H} \phi M \phi^{*} H^{*} \\
\mathcal{N} & =[\mathrm{I}+\mathrm{H} \phi \mathcal{K}] \mathcal{D}[\mathrm{I}+\mathrm{H} \phi \mathcal{K}]^{*} \\
{[\mathrm{I}+\mathrm{H} \phi \mathcal{K}]^{-1} } & =[\mathrm{I}-\mathrm{H} \psi \mathcal{K}] \\
\mathcal{M}^{-1} & =[\mathrm{I}-\mathrm{H} \psi \mathcal{K}]^{*} \mathcal{D}^{-1}[\mathrm{I}-\mathrm{H} \psi \mathcal{K}]
\end{aligned}
$$

The first expression defines the Newton-Euler operator factorization of the mass matrix $\mathcal{M}$ in terms of the $\mathrm{H}$ hinge articulation, the $\phi$ rigid body propagation and the $M$ link spatial inertia operators. While this factorization has nonsquare factors, the second expression describes an alternative factorization involving only square factors with block diagonal $\mathcal{D}$ and block lower-triangular $[\mathrm{I}+\mathrm{H} \phi \mathcal{K}]$ matrices. This factorization involves new spatial operators that are associated with the articulated body $(A B)$ forward dynamics algorithm [4, 5] for the system. The next expression describes an analytical expression for the inverse of the $[\mathrm{I}+\mathrm{H} \phi \mathcal{K}]$ operator. Using this leads to the final analytical expression for the inverse of the mass matrix. These operator expressions hold generally for tree-topology systems irrespective of the number of bodies, the types of hinges, the specific topological structure, and even for non-rigid links [6].

\section{B. The $\Omega$ extended operational space compliance matrix}

With $\mathcal{V} \in \mathcal{R}^{6 n}$ denoting the stacked vector of link spatial velocities, its spatial operator expression is [6]

$$
\nu=\phi^{*} H^{*} \dot{\theta}
$$

Bundling together the rigid body transformations for all nodes we define the $\mathcal{B} \in \mathcal{R}^{6 n_{c} \times 6 n}$ pick-off matrix such that

$$
V_{c}=\mathcal{B V} \stackrel{42}{=} \mathcal{B} \phi^{*} H^{*} \dot{\theta} \Rightarrow \mathcal{J} \underline{\underline{3}} \mathcal{B} \phi^{*} H^{*}
$$

This is the spatial operator expression for the $\mathcal{f}$ Jacobian matrix. Using this expression and Eq. 41 for the mass matrix inverse within Eq. 6 leads to the following expression for $\underline{\Lambda}$ :

$$
\begin{aligned}
& \underline{\underline{\underline{6}}} \partial \mathcal{M}^{-1} \partial^{*} \\
& \stackrel{41}{=} \mathcal{B}^{*} \phi^{*} \mathrm{H}^{*}(\mathrm{I}-\mathrm{H} \psi \mathcal{K})^{*} \mathcal{D}^{-1}(\mathrm{I}-\mathrm{H} \psi \mathcal{K}) \mathrm{H} \phi \mathcal{B}
\end{aligned}
$$

Using the following spatial operator identity from $[6,10]$

$$
(\mathrm{I}-\mathrm{H} \psi \mathcal{K}) \mathrm{H} \phi=\mathrm{H} \psi
$$

in Eq. 44 leads to the following simpler expression for $\underline{\Lambda}$ :

$$
\underline{\Lambda}=\mathcal{B}^{*} \Omega \mathcal{B} \text { with } \Omega \triangleq \psi^{*} H^{*} \mathcal{D}^{-1} H \psi \in \mathcal{R}^{6 n_{\mathrm{c}} \times 6 n_{\mathrm{c}}}
$$

We have arrived at an expression for $\underline{\Lambda}$, that unlike Eq. 6 , involves neither the mass matrix inverse nor the node's Jacobian matrix! We refer to $\Omega$ as the extended operational space compliance matrix. This terminology is based on Eq. 46 which shows that the OSCM, $\underline{\Lambda}$ can be obtained by a reducing transformation of the full, all body $\Omega$ matrix by the $\mathcal{B}$ pick-off operator involving just the matrix sub-blocks associated with the parent links of the nodes. From its definition, it is clear that $\Omega$ is a symmetric and positive semi-definite since $\mathcal{D}^{-1}$ is a symmetric positive-definite matrix.

While the explicit computation of $\mathcal{M}^{-1}$ or $\mathcal{J}$ is not needed to obtain $\Lambda$, the direct evaluation of Eq. 46 still remains of $\mathrm{O}\left(\mathcal{N}^{3}\right)$ complexity due to the need for carrying out the multiple matrix/matrix products. The next section shows that these matrix/matrix products can be avoided by exploiting a decomposition of the $\Omega$ matrix.

\section{Decomposition of $\Omega$}

The following lemma describes a decomposition of $\Omega$ into simpler component terms and an expression for its block elements. The $\mathcal{E}_{\psi}^{*}$ and $\psi()$ terms used below are defined in references [5, 6]. Furthermore, $\wp(k)$ denotes the parent link for the $k^{\text {th }}$ link, and $i \prec j$ notation implies that the $j^{\text {th }}$ link is an ancestor of the $i^{\text {th }}$ link in the tree.

\section{Lemma 1 Decomposition of $\Omega$}

$\Omega$ can be decomposed into the following disjoint sum:

$$
\begin{gathered}
\Omega=\Upsilon+\tilde{\psi}^{*} \Upsilon+\Upsilon \tilde{\psi}+R \\
\text { where } R \triangleq \sum_{\substack{\forall i, j: i \nless \neq j \\
k=\wp(i, j)}} e_{i} \psi^{*}(k, i) Y(k) \psi(k, j) e_{j}^{*}
\end{gathered}
$$

$\Upsilon \in \mathcal{R}^{6 n_{\mathrm{c}} \times 6 \mathrm{n}_{\mathrm{c}}}$ is a block-diagonal operator, referred to as the operational space compliance kernel, satisfying the following backward Lyapunov equation:

$$
\mathrm{H}^{*} \mathcal{D}^{-1} \mathrm{H}=\Upsilon-\operatorname{diagOf}\left\{\mathcal{E}_{\psi}^{*} \Upsilon \varepsilon_{\psi}\right\}
$$

diagOf $\left\{\mathcal{E}_{\psi}^{*} \curlyvee \varepsilon_{\psi}\right\}$ represents just the block-diagonal part of the (generally non block-diagonal) $\mathcal{E}_{\psi}^{*} \curlyvee \mathcal{E}_{\psi}$ matrix. The $6 \times 6$ dimensional, symmetric, positive semi-definite $\Upsilon(\mathrm{k})$ diagonal matrices satisfy the following parent/child recursive relationship:

$\Upsilon(k)=\psi^{*}(\wp(k), k) \Upsilon(\wp(k)) \psi(\wp(k), k)+H^{*}(k) \mathcal{D}^{-1}(k) H(k)$

This relationship forms the basis for the following $\mathrm{O}(\mathcal{N})$ baseto-tips scatter recursion for computing the $\Upsilon(\mathrm{k})$ diagonal elements:

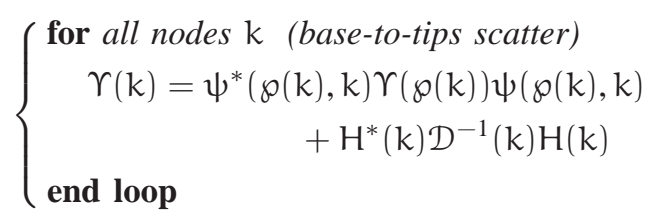

While $\gamma$ defines the block-diagonal elements of $\Omega$, the following recursive expressions describe its off-diagonal terms:

$$
\Omega(i, j)= \begin{cases}\Upsilon(i) & \text { for } i=j \\ \Omega(i, k) \psi(k, j) & \text { for } i \succeq k \succ j, \quad k=\wp(j) \\ \Omega^{*}(\mathfrak{j}, \mathbf{i}) & \text { for } i \prec j \\ \Omega(i, k) \psi(k, j) & \text { for } i \nsucc j, j \neq i, k=\wp(i, j)\end{cases}
$$

Proof: See [5, 6]. 
Eq. 47 shows that $\Omega$ can be decomposed into the sum of simpler terms consisting of the block diagonal $\Upsilon$, the uppertriangular $\tilde{\psi}^{*} \Upsilon$, the lower triangular $\Upsilon \tilde{\psi}$, and the sparse $R$ matrices. Furthermore, Eq. 51 reveals that all of the blockelements of $\Omega(i, j)$ can be obtained from the $\Upsilon(i)$ elements of the $\Upsilon$ block-diagonal operational space compliance kernel. Since only a small subset of the elements of $\Omega$ are needed for computing $\Lambda$, the next section exploits this to avoid the expensive computation of the full $\Omega$ matrix.

\section{Computing $\Lambda$}

From the $\Lambda=\mathcal{B}^{*} \Omega \mathcal{B}$ expression, and the sparse structure of $\mathcal{B}$, it is clear that only a subset of the elements of $\Omega$ are needed to compute $\underline{\Lambda}$. The $\mathcal{B}$ pick-off operator has one column for each of the nodes, with each such column having only a single non-zero $6 \times 6$ matrix entry at the $k^{\text {th }}$ parent link slot. Only as many elements of $\Omega$ as there are elements in $\Lambda$ are needed. Thus, just $n_{c} \times n_{c}$ number of $6 \times 6$ sub-block matrices of $\Omega$ are required. In view of the symmetry of the matrices, we actually need just $n_{c}\left(n_{c}+1\right) / 2$ such sub-block matrices. The overall cost of this algorithm is linearly proportional to the number of degrees of freedom, and a quadratic function of the number of nodes. This is much lower than the $\mathrm{O}\left(\mathcal{N}^{3}\right)$ cost implied by Eq. 6.

\section{E. Simulation example}

We use a simulation of a multi-link pendulum colliding with itself and the environment to measure the performance of our minimal coordinate formulation. The environment consists of a floor and a wall located $4 \mathrm{~m}$ away. The multi-link pendulum consists of $\mathrm{n}$ identical $1 \mathrm{~kg}$ mass spherical bodies connected with pin hinges. The radius of the sphere is scaled based on the number of links to maintain a $12 \mathrm{~m}$ overall length of the pendulum. The pendulum base is located at a height of $10 \mathrm{~m}$. The open source Bullet software [1] is used for collision detection, and the PATH software [2] for solving mixed complementarity problems.

The simulation lasts for 5 seconds with a time step of $0.1 \mathrm{~ms}$. A coefficient of restitution of 0.5 is used to simulate inelastic collisions. The pendulum starts at an angle of $\pi / 2$ radians with an initial angular velocity of 1radian/s and a gravitational acceleration of $9.8 \mathrm{~m} / \mathrm{s}^{2}$.

As the pendulum swings from left to right, it collides with the ground, bounces off of the ground, and eventually collides with the wall on the right. In the course of the sequence, multiple links are at times in collision with the ground, the wall and with each other as seen in Figure 1 for a 12-link pendulum.

We simulate the contact and collision dynamics using two different techniques. In the first minimal coordinate $(M C)$ technique, the serial-chain pendulum is modeled using minimal coordinates and only unilateral contact constraints. The second redundant coordinate $(R C)$ technique uses non-minimal coordinates where each link is treated as an independent body, and the hinges are handled as bilateral constraints between the neighboring links. We have verified good agreement between
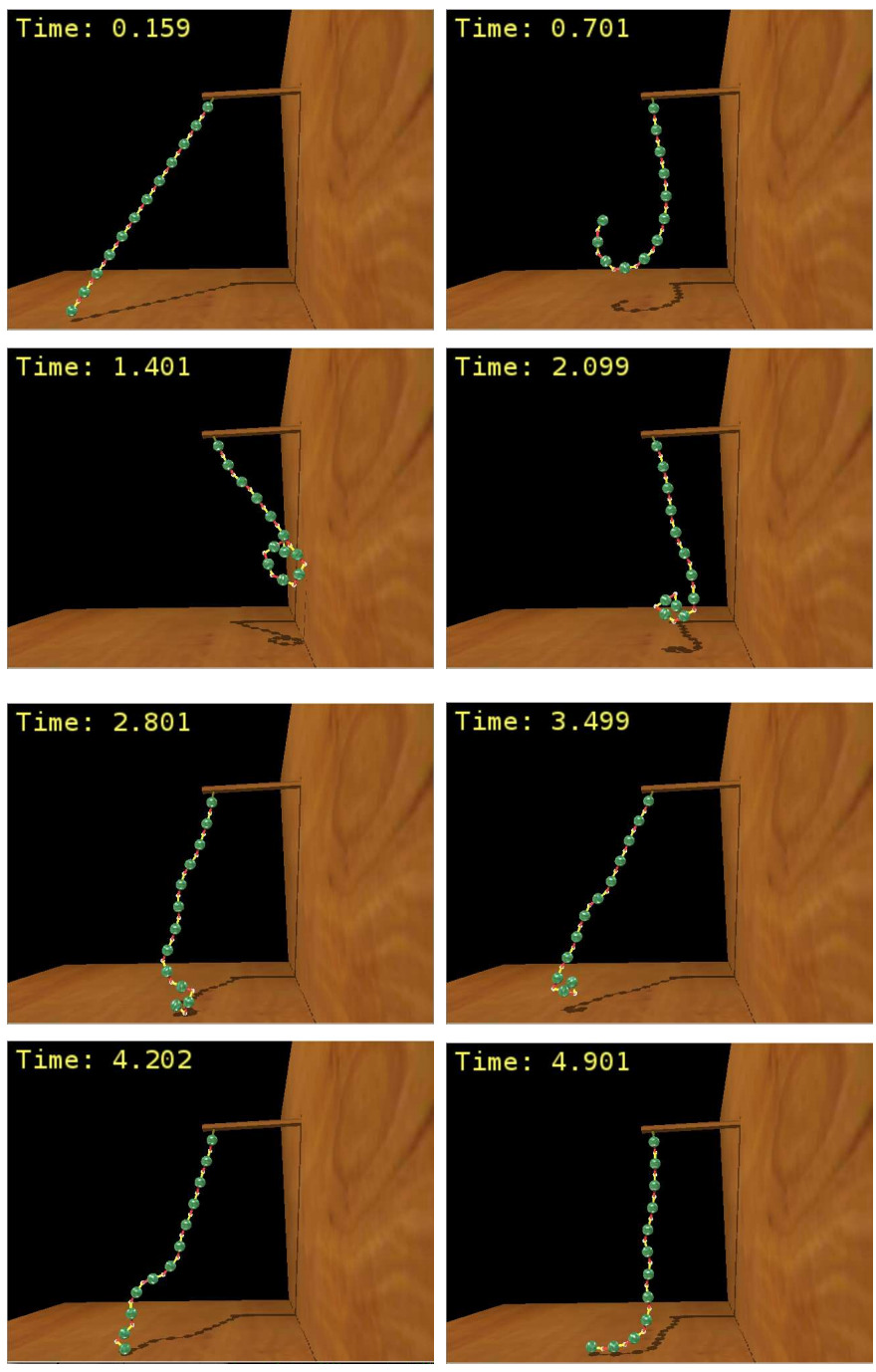

Fig. 1. Time series capture of swinging pendulum simulation with 12 links

the solutions from these two methods, Figure 2 shows the similar time history of the height and normal velocity of the
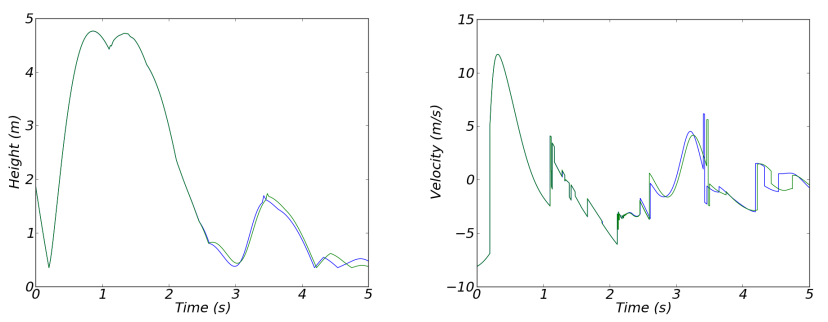

Fig. 2. Comparisons of the height and normal velocity of the last link using the $\mathrm{MC}$ and $\mathrm{RC}$ methods for a 12-body pendulum

last link of a 12-body pendulum from the two methods.

Table I compares the computational cost of the $\mathrm{MC}$ and $\mathrm{RC}$ methods for pendulums containing 3 to 30 links. We observe that the MC method is 3 to 50 times computationally faster than the RC method. Moreover the performance gap widens 


\begin{tabular}{|c|c|c|}
\hline & \multicolumn{2}{|c|}{ Computation Time (s) } \\
\hline Number of Bodies & Minimal Coords & Redundant Coords \\
\hline 3 & 13.3258 & 47.7948 \\
6 & 10.5026 & 68.7122 \\
12 & 19.0632 & 305.2764 \\
15 & 21.7605 & 558.0966 \\
24 & 38.1993 & 1899.8575 \\
30 & 73.7386 & 4100.5061 \\
\hline
\end{tabular}

TABLE I

A comparison of the computational time for the minimal coordinate $(M C)$ and the redundant coordinate $(R C)$ techniques for the multi-link pendulum example with different number of links.

substantially as the number of links in the system is increased.

\section{Vi. CONCLusions}

In this article we have described the formulation of the contact and collision dynamics for multi-link systems using minimal coordinates. With minimal coordinates, the size of the LCP problem in Eq. 34 is $\left(n_{\mathfrak{u}}\left(n_{f}+2\right)+n_{b}\right)$ which is independent of the number of links and the number of degrees of freedom in the system. In contrast, the size of the corresponding LCP problem using redundant coordinates has dimension larger by $6 n-\mathcal{N}$ or more depending on the specific formulation. For a 6-link manipulator with 6 degrees of freedom, this amounts to dimensional difference of 30 . The use of minimal coordinates also results in the automatic enforcement of the bilateral constraints such as from interlink hinges and avoids the need for DAE type error control schemes for inter-link hinge bilateral constraints.

We have described the variants of the LCP problem needed to handle effects such as bilateral constraints associated with closed-chain topologies as well as elastic and inelastic collision dynamics. Our treatment of unilateral constraints allows us to handle them in the same was as bilateral constraints in the operational space based formulation. Finally, we have shown that existing low order computational algorithms for computing the OSCM can be used to make the computation of the LCP matrices tractable. We have used a multi-link pendulum contact and collision dynamics simulation example to illustrate the computational speedup achieved by the minimal coordinate approach.

\section{Acknowledgments}

The research described in this paper was performed at the Jet Propulsion Laboratory (JPL), California Institute of Technology, under contract with the National Aeronautics and Space Administration $\sqrt{3}^{3}$ This project was also supported in part by the DARPA Autonomous Robotic Manipulation Software Track (ARM-S) program.

\footnotetext{
${ }^{3}$ (c) 2012 California Institute of Technology. Government sponsorship acknowledged.
}

\section{REFERENCES}

[1] Bullet Physics Library, $2012 . \quad$ URL http://bulletphysics.org.

[2] The PATH Solver, 2012. URL http://pages.cs.wisc.edu/ ferris/path.html.

[3] M Anitescu and F A Potra. Formulating dynamic multirigid-body contact problems with friction as solvable linear complementarity problems. Nonlinear Dynamics, 14(3):231-247, 1997.

[4] Roy Featherstone. Rigid Body Dynamics Algorithms. Springer Verlag, 2008.

[5] Abhinandan Jain. Graph Theoretic Foundations of Multibody Dynamics Part II: Analysis and Algorithms. Multibody System Dynamics, 26(3):335-365, October 2011.

[6] Abhinandan Jain. Robot and Multibody Dynamics: Analysis and Algorithms. Springer, 2011.

[7] Oussama Khatib. A Unified Approach for Motion and Force Control of Robot Manipulators: The Operational Space Formulation. IEEE Journal of Robotics and Automation, RA-3(1):43-53, February 1987.

[8] K Kreutz-Delgado, Abhinandan Jain, and Guillermo Rodriguez. Recursive formulation of operational space control. Internat. J. Robotics Res., 11(4):320-328, 1992.

[9] F Pfeiffer. Mechanical System Dynamics. Springer, 2005.

[10] Guillermo Rodriguez, Abhinandan Jain, and K KreutzDelgado. A spatial operator algebra for manipulator modeling and control. Internat. J. Robotics Res., 10(4): 371, 1991.

[11] D. Stewart and Jeffrey C Trinkle. An implicit time-stepping scheme for rigid body dynamics with Coulomb friction. In Proceedings 2000 ICRA. Millennium Conference. IEEE International Conference on Robotics and Automation. Symposia Proceedings (Cat. No.00CH37065), pages 162-169. Ieee, 2000.

[12] A Tasora and M Anitescu. A matrix-free cone complementarity approach for solving large-scale, nonsmooth, rigid body dynamics. Computer Methods in Applied Mechanics and Engineering, 200(5-8):439-453, January 2011.

[13] Jeffrey C Trinkle. Formulation of Multibody Dynamics as Complementarity Problems. In ASME International Design Engineering Technical Conference, Chicago, IL, September 2003.

[14] Katsu Yamane and Yoshihiko Nakamura. A Numerically Robust LCP Solver for Simulating Articulated Rigid Bodies in Contact. In Robotics: Science and Systems IV, chapter A numerica, pages 89-104. MIT Press, 2009. 